However, should there be doubt about whether or not the facial nerve has been cut then the presence of these action potentials gives immediate assurance that the nerve is in continuity.

Gilliatt and Taylor (1959) measured conduction time in the facial nerve between the stylomastoid foramen and orbicularis oculi in three patients before and after complete nerve section. They found no change in the latency of the muscle response but noticed a sudden diminution of amplitude after the third post-operative day, together with disappearance of the visible twitch. In a further four patients whose facial nerves were completely sectioned during removal of acoustic neuromata modified intensity-duration curves were plotted from orbicularis oculi at daily intervals. The results of this test showed an increasing threshold to 0.1 and 1 millisecond stimuli from the fourth post-operative day. Our experience has been that, whereas nerve-excitability tests usually give a clear indication and require little interpretation immediately after the third day, intensityduration curves may alter insidiously at first. Thus we feel simplicity of apparatus, performance, and interpretation commend the use of nerve-excitability tests in facial palsy.

The practical application of nerve excitability tests is in the assessment of treatment. Widely different measures have been advised in the treatment of Bell's palsy, such as decompression, the use of cortisone derivatives, and cervical sympathetic block. The efficacy of these or any other methods cannot be assessed unless the number of patients with physiological block are known, for $90 \%$ of these will achieve full recovery regardless of treatment.

\section{Summary}

A simple test of motor-nerve excitability is described. This test distinguishes between physiological block and degeneration of the facial nerve as early as 72 hours after the onset of facial palsy.

The results of this test and their relation to the time and degree of recovery in facial-nerve palsies of various types are described.

It is suggested that such a test should be used in the assessment of treatments of facial palsy.

We thank all those physicians and surgeons who have referred cases to us, especially Miss J. Collier and Dr. H. Dimsdale for their help, interest, and advice.

\section{REFERENCES}

Adams, R. D., Denny-Brown, D., and Pearson, C. M. (1953). Diseases of Muscle, p. 101. Cassell, London.

Campbell, E. D. R. (1954). Brit. J. phys. Med., 17, 215.

Collier, J. (1959). Proc, roy, Soc, Med., 52, 1075.

Duchenne, G. B. (1872). De l'Electrisation Localisée, 3rd ed. Baillière, Paris.

Gilliatt, R. W., and Taylor, J. C. (1959). Proc. roy. Soc. Med. 52, 1080

Laudau, W. M. (1953). J. Neurosurg., 10, 64

Richardson, A. T., and Wynn Parry, C. B. (1957). Ann. phys. Med., 4. 3,41 .

Taverner, D. (1955). Brain, 78, 209.

\title{
URINARY INFECTION AND ANAEMIA IN PREGNANCY
}

\author{
C. GILES, M.D., B.Sc. \\ BY \\ From the Department of Pathology, City General Hospital, Stoke-on-Trent
}

Two of the most common causes of anaemia in pregnancy are iron and folic-acid deficiency. A significant proportion of patients, however, who are receiving these haematinics remain anaemic or become anaemic as pregnancy progresses. This has been observed in the antenatal clinic of the City General Hospital, Stoke-on-Trent, where both iron and folic acid have been given for a number of years (Giles and Burton, 1960). Anaemia in these cases might be due to excessive haemodilution (Dieckmann and Wegner, 1934), failure on the part of the patient to take her drugs, or possibly to some other factor such as intercurrent infection. Since the urinary tract is by far the commonest site of infection in pregnancy, an attempt has been made over the past two years to determine whether urinary infection is related to some of these cases of refractory anaemia.

Any disorder in late pregnancy, whether it be anaemia, urinary infection, or both, must be treated without delay. Elaborate investigations or controlled therapeutic trials are therefore not usually practicable, and it was apparent that any study of this problem would have to be based on relatively simple tests. The accepted criteria of urinary infection have been based by some authors (Stansfeld, 1954; Hutt et al., 1961) on finding an excess of leucocytes in measured specimens of urine and by others (Kass, 1957 ; Turner, 1961) on cultural methods or on the more reliable but time-consuming " pyrexal" test (Pears and Houghton, 1959 ; Hutt et al., 1961). In the present investigation, carried out on large numbers of out-patients, a simple combination of some of these methods was adopted, using mid-stream rather than catheter specimens, as the latter were thought to be too dangerous (Kass, 1957).

\section{Methods}

All patients attending the hospital antenatal clinic received prophylactic iron by mouth after their first visit and routine folic acid from the twenty-eighth week of pregnancy until after the confinement. Women in whom a haemoglobin below $70 \%(10.4 \mathrm{~g} . / 100 \mathrm{ml}$.) had been observed on more than one occasion were included in the present investigation. A brief case-history was taken, the haemoglobin and mean corpuscular haemoglobin concentration (M.C.H.C.) were determined, and a stained blood film was examined. A mid-stream specimen of urine was obtained with suitable precautions, and where it was thought advisable a specimen of blood was taken for serum iron, serum vitamin $\mathbf{B}_{12}$, and, latterly, for serum folic-acid estimation. The bone-marrow was examined by sternal puncture in a number of cases.

Urine specimens were cultured, using standard loopfuls, on blood and MacConkey agar; a portion of the specimen was then set aside for leucocyte count and the remainder centrifuged. The supernatant was examined for protein and the deposit for the presence of casts, red cells, and leucocytes. In all cases where the leucocytes exceeded 5 per high-power field a leucocyte count was performed on the portion of the specimen previously set aside for this purpose. Counts of $25,000 / \mathrm{ml}$. or more were considered significant ; in severe infections the count amounted to several million leucocytes per $\mathrm{ml}$. Cultures were regarded as 
positive if more than five colonies of a pathogenic organism were isolated after 18 to 24 hours' incubation. Whenever possible, cases of pyuria were re-examined at the next attendance and the haematological examinations repeated at frequent intervals until after the confinement. In cases of established urinary infection treatment with suitable antibacterial drugs was advised according to the in-vitro sensitivity of the organism concerned. The drugs most commonly used were triple sulphonamide, nitrofurantoin, a combination of penicillin and streptomycin, and sometimes tetracycline.

In order to establish the incidence of urinary infection in a large group of pregnant women who were not anaemic, patients attending the antenatal clinic between the thirty-second and thirty-fourth weeks of pregnancy were taken at random and used as controls. A mid-stream specimen of urine from each of these cases was examined and the haemoglobin estimated at the same time. Most of this latter group of patients were, of course, not anaemic, but a certain overlap in haemoglobin levels between patients and controls was observed. The two groups were therefore considered together when calculating the incidence of urinary infection at different haemoglobin levels.

\section{Results}

Incidence of Urinary Infection

Altogether 910 cases were available for study; of these, 463 were cases of "anaemia" and 447 were controls whose haemoglobin was not known beforehand. Cases may be conveniently divided into four categories

Table I.-Urinary Findings in Patients, Classified According to the Haemoglobin Level (Haemoglobin: $100 \%=14.8 \mathrm{~g} . / 100 \mathrm{ml}$.)

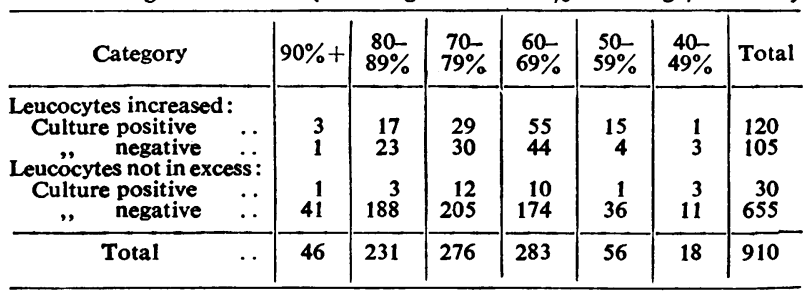

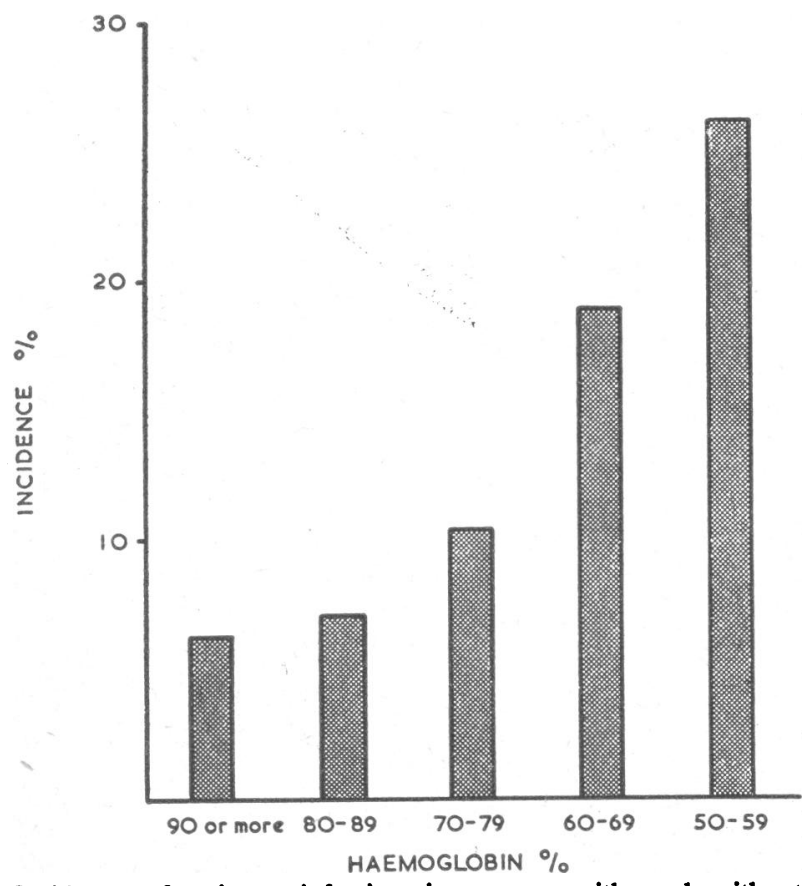

Incidence of urinary infection in women with and without anaemia. -namely, those with and without pus and those with and without positive cultures. Only cases in which both criteria were positive have been classified as true urinary infections. These totalled 120 cases, of which 91 were derived from the group of anaemic patients who were studied in more detail.

A similar number of women showed the presence of pus with a negative culture. While it is possible that in some of these the pus originated in the urinary tract, it seems more likely that in the majority it came from the vagina. A high incidence of trichomonal infestation observed in this series lends support to this assumption, and on several occasions catheter specimens, which were subsequently taken from patients in this category, were found to be normal.

A much smaller number presented without pus but with positive cultures. This small group has been ignored in the present study. The incidence of "true" urinary infection is illustrated in the Chart.

The increased incidence of urinary infection in patients with haemoglobin levels below $80 \%(11.8 \mathrm{~g} . / 100 \mathrm{ml}$.$) is$ shown, reaching its maximum at haemoglobins between 50 and $59 \%$. Infection was present in $7.2 \%$ of cases with "normal" haemoglobins, and this contrasts sharply with an incidence of $15.8 \%$ in cases of anaemia. The difference is statistically highly significant $\left(\chi^{2}=\right.$ $11.68 ; \mathrm{P}<0.001$ ).

An analysis follows of the 91 cases of urinary infection which occurred in patients with pregnancy anaemia.

\section{Iron and Vitamin Deficiencies}

Some degree of iron deficiency, as shown by hypochromia and a M.C.H.C. below $31 \%$, confirmed in many cases by serum iron estimations, was present in $57 \%$ of cases of anaemia with urinary infection. Folicacid deficiency was not commonly observed and occurred only in women who had not received any prophylactic folic acid. One, a primigravida aged 24 , was found to have a frank megaloblastic marrow; two others had low serum folic-acid levels, one of them a twin pregnancy.

All the serum vitamin $B_{12}$ estimations carried out in this series were within the normal range.

\section{Bacteriological Findings}

Table II lists the different pathogenic organisms isolated from the urine of patients under discussion. As observed by other workers (Pinkerton et al., 1961 ; Turner, 1961), E. coli appeared to be by far the commonest cause of urinary infection, having been isolated in $74 \%$ of cases. Str. faecalis, Staph. aureus, and $P r$. vulgaris were next in order of frequency.

\begin{tabular}{|c|c|c|c|}
\hline Organism & $\begin{array}{l}\text { No. of } \\
\text { Cases }\end{array}$ & Organism & $\begin{array}{l}\text { No. of } \\
\text { Cases }\end{array}$ \\
\hline $\begin{array}{l}\text { Escherichia coli } \\
\text { Streptococcus faecalis } \\
\text { Staphylococcus aureus } \\
\text { Proteus vulgaris .. }\end{array}$ & \begin{tabular}{rr|}
$\cdots$ & 61 \\
$\cdots$ & 12 \\
$\cdots$ & 5 \\
$\cdots$ & 4
\end{tabular} & $\begin{array}{l}\text { E. coli and Pr. vulgaris } \\
\text { K’lebsielläaerogenes } \\
\text { Pseudomonas pyocyanea }\end{array}$ & $\begin{array}{l}\cdots \\
\cdots \\
\cdots\end{array}$ \\
\hline
\end{tabular}

Response to Treatment

The response to therapy was examined in 56 cases, and in 33 of these the haemoglobin rose by $10 \%$ or more prior to confinement. Table III shows that 30 cases presented with one or more clear urine specimeas after a minimum of two weeks' antibacterial therapy. In 26 of these treatment of the infection was followed by a rise in their haemoglobin level by $10 \%$ or more, while four remained anaemic as long as they were under 
observation. However, in the group of 26 whose urine remained infected, a significant improvement of the anaemia occurred in only 7, while in 19 the haemoglobin remained at or near the original level.

$$
\begin{aligned}
& \text { TABLE III.-Response to Therapy } \\
& \text { Category }
\end{aligned}
$$

In many instances it was difficult to decide whether the improvement of the anaemia resulted from the elimination of infection or from more intensive iron therapy. In 18 women who first attended the clinic late in pregnancy iron had been taken only sporadically or not at all. The remaining patients gave a history of regular prophylactic treatment with both iron and folic acid. It is possible, however, that some of them became more alert to the need of regularly taking their haematinic drugs, as a result of a more personal approach during the period of investigation. Parenteral in addition to oral iron was given to eight patients, and in all but one case was followed by a rapid rise of the haemoglobin level.

Analysis of Table III reveals a number of paradoxical results: in seven cases the anaemia was corrected, though urinary infection persisted, and, conversely, four patients remained anaemic, although the urine became clear after antibacterial treatment.

Of the original 91 cases, 35 were not included here, either because urinary infection was diagnosed too late in pregnancy for treatment to be effective or because patients defaulted.

\section{Assessment of Renal Function}

Urinary infection persisted after several weeks' treatment in some patients, 13 of whom were admitted for renal investigation. A combined renal function test, which incorporates urea clearance and concentration along with hourly specific gravity measurements and total urea excretion, was used. This test produced normal results in 10 out of the 13 cases.

\section{Clinical Findings}

In most cases urinary infection was symptomless. Only nine patients $(10 \%)$ presented with the classical signs and symptoms of pyelitis; in a further seven a history of dysuria and suprapubic pain could be obtained on direct questioning. Very many women in late pregnancy complained of backache and frequency of micturition, but in the great majority the urine was quite normal.

In 10 patients there was a history of infection of the urinary tract in previous pregnancies, and three of these were included twice in the present series with urinary infection in successive pregnancies.

Of the 463 women with anaemia who were investigated, 117 were primigravidae (Table IV). The incidence of urinary infection in these patients was $30.8 \%$, as compared with $15.9 \%$ in multigravidae. These findings agree with those of Pinkerton et al. (1961). Despite the higher incidence, primigravidae tended to respond to the treatment of infection rather better than women who had already borne children.

As urinary infection was asymptomatic in the majority of cases it is impossible to say how long it had been present when it was recognized. Table $\mathrm{V}$ shows that in only 11 cases the infection was diagnosed before the

\begin{tabular}{|c|c|c|c|c|c|c|}
\hline $\begin{array}{l}\text { Weeks of gestation } \\
\text { No. of cases } \quad . .\end{array}$ & $16-15$ & $\frac{20-23}{10}$ & $24-27$ & $\begin{array}{c}28-31 \\
28\end{array}$ & $\frac{32-35}{26}$ & $\underset{12}{36-39}$ \\
\hline
\end{tabular}
twenty-fourth week. A study of the medical case sheets revealed that many patients entered their pregnancy with a reasonably normal haemoglobin, which began to fall some three to six weeks before the presence of anaemia led to the discovery of their urinary infection.

Table IV.-Distribution of Cases According to Parity

\begin{tabular}{c|c|c}
\hline Parity & No. Infected & Total No. \\
\hline 1 & 36 & 117 \\
2 & 16 & 95 \\
3 & 13 & 95 \\
4 & 4 & 38 \\
5 & 8 & 38 \\
6 & 4 & 34 \\
7 & 4 & 17 \\
8 to 14 & 2 & 29 \\
Not known & & \\
\hline
\end{tabular}

TABle V.-Diagnosis of Urinary Infection in Relation to Period of Gestation

\section{Discussion}

The present study has shown quite clearly that there is an association between urinary infection and anaemia in pregnancy. Since both are among the commonest complications of pregnancy, the question arises whether the two disorders are causally related, and whether urinary infection predisposes to anaemia or vice versa. As there is little evidence that slight or moderate degrees of anaemia render the body more liable to infection, it seems likely that anaemia develops as a result of infection of the urinary tract. It is therefore of interest to consider the possible mechanisms by which this may be brought about. As is generally accepted, refractory anaemia appears in the course of advanced chronic pyelonephritis. This has been explained in terms of a diminished output of erythropoietic hormone caused by severe renal damage (Gurney et al., 1957 ; Gallagher et al., 1959; Penington, 1961). The results of the present study do not suggest that anaemia in pregnant women with urinary infections is due to lack of erythropoietin. Renal damage could not be demonstrated in the majority of patients with refractory infection of the urinary tract. Moreover, renal lesions are more likely to result from recurrent infection than from a single attack, and a higher incidence of urinary infection with anaemia might be expected in multigravidae. Observations have shown that this was not the case in the present series: primigravidae were nearly twice as often affected as multigravidae.

The mechanism of the accompanying anaemia is not clear. Iron deficiency was present in well over $50 \%$ of patients, and it is possible that infection is responsible for this, either by interfering with the absorption of this metal or by giving rise to disordered iron metabolism, as suggested by Guggisberg (1951). On the other hand, it may be argued that iron deficiency is completely unrelated to the infection and merely reflects the patient's failure to "take her tablets." This was certainly the case in a number of instances; most women, however, maintained that they had regularly taken both iron and folic acid, as prescribed.

If iron deficiency resulted from urinary infection an increase in haemoglobin levels might be expected after antibacterial therapy. In the present investigation a satisfactory rise in the haemoglobin occurred more 
often when antibacterial treatment was successful than in patients where urinary infection persisted. Unfortunately, it was not always possible to decide whether the improvement of the anaemia resulted from the elimination of infection or from more intensive iron therapy.

While the significance of iron deficiency in these cases remained obscure, there was little evidence to suggest that folic-acid deficiency played a relevant part in the causation of their anaemia. This might have been anticipated, as most of them were on a prophylactic regimen which includes folic acid. Although pregnant women are often reluctant to take iron, few default on their folic acid.

Only 16 patients presented with the clinical signs and symptoms of pyelitis or cystitis. In $82 \%$ the infection was quite symptomless. Routine laboratory investigation of the urine in pregnancy would therefore appear to be advisable, especially at the beginning of the third trimester. Many cases of urinary infection in pregnancy are undoubtedly missed or erroneously diagnosed as "albuminuria," as a result of simple side-room testing. Urinary infection should be suspected in all cases of anaemia which have not responded to routine iron and folic-acid therapy, and once diagnosed should be treated promptly and energetically.

\section{Summary}

The incidence of urinary infection has been determined in 463 cases of anaemia in pregnancy; they were compared with 447 normal pregnant women. The great majority of patients and controls had been receiving routine prophylactic iron and folic acid.

Urinary infection was more than twice as common in the anaemic patients as in the controls. In 30 out of 56 cases the infection cleared on antibacterial drug therapy and in most of these the anaemia also responded.

Evidence of iron deficiency was present in more than half of the anaemias with urinary infection, but folicacid deficiency appeared to be uncommon in these cases.

This investigation was made possible only by the kind co-operation of Mr. H. Burton and Mr. L. M. Edwards, and by the help of the Research Subcommittee of the Birmingham Regional Hospital Board, which provided technical assistance and necessary equipment.

We wish to thank the whole staff of the antenatal clinic and the pathology department of the City General Hospital, Stoke-on-Trent, and in particular Miss Kathleen Weaver and Miss Anne Archer, for their untiring help and patience in dealing with most of the patients and for carrying out vitamin-B ${ }_{12}$ estimations; Dr. E. W. Ball for the folic-acid estimations; Miss M. R. Haslock for the serum iron determinations ; and Mr. A. H. Iliffe, of the University of North Staffordshire, for help with the statistical analysis.

\section{REFERENCES}

Dieckmann, W. J., and Wegner, C. R. (1934). Arch. intern. Med., 53, 71, 188, 345, 353.

Gallagher, N. I., McCarthy, J. M., Hart, K. T., and Lange, R. D. (1959). Blood, 14, 662.

Giles, C., and Burton, H. (1960). Brit. med. J., 2, 636

Guggisberg, H. (1951). Zbl. Gynäk., 73, 452.

Gurney, C. W., Goldwasser, E., and Pan, C. (1957). J. Lab. clin. Med., $50,534$.

Hutt, M. S. R., Chalmers, J. A., McDonald, J. S., and de Wardener, H. E. (1961), Lancet, 1, 351.

Kass, E. H. (1957). Arch. intern. Med., 100, 709.

Pears, M. A., and Houghton, B. J. (1959). Lancet, 2, 1167.

Penington, D. G. (1961). Ibid., 1, 301.

Pinkerton, J. H. M., Wood, C., Williams, E. R., and Calman, R. M. (1961). Brit. med. J., 2, 539.

Stansfeld, J. M. (1954). Proc. roy. Soc. Med., 47, 631.

Turner, G. C. (1961). Lancet, 2, 1062.

\section{PREVENTION OF CATHETER INFECTION OF URINE IN FEMALE PATIENTS}

BY

W. A. GILLESPIE, M.A., M.D., F.R.C.P.I., D.P.H. Professor of Clinical Bacteriology, University of Bristol G. G. LENNON, M.B., Ch.M., F.R.C.O.G.

Professor of Obstetrics and Gynaecology, University of Bristol

K. B. LINTON, Ph.D., B.Sc.*

Research Bacteriologist, United Bristol Hospitals

AND

N. SLADE, M.B., F.R.C.S.

Consultant Urologist, Southmead General Hospital, Bristol

Open drainage of the male or female bladder by indwelling catheter is very apt to cause urinary infection. Intermittent catheterization carries a smaller but still appreciable risk. Even the simple passage of a sterile catheter may introduce organisms from the introitus or urethra into the bladder. Kass (1957) reported an infection rate of $2-4 \%$ after a single catheterization. Slade and Linton (1960) reported an infection rate of $29 \%$ in obstetric patients who were catheterized once for therapeutic reasons. Brumfitt et al. (1961) also found a high infection rate $(22.8 \%)$ in obstetric patients who required therapeutic catheterization because of complicated or difficult labour, but a lower rate $(9.1 \%)$ in patients who were catheterized solely to obtain urine for examination. The risk of infection is increased with repeated catheterization or with an abnormal urinary tract. The danger of catheter-induced infection should be avoided by using mid-stream specimens of urine when possible instead of catheter specimens for diagnostic purposes in female patients as well as males (British Medical Journal, 1961). When catheterization is essential the risk of infection should be reduced by employing a correct aseptic technique. In this paper we report trials of two methods of further reducing the risk by the prophylactic use of disinfectants. In one method the urethra was disinfected before passing the catheter. In the other the bladder was disinfected after catheterization.

The laboratory methods and the criteria for the diagnosis of infection were described by Gillespie $e t$ al. (1960) and by Slade and Linton (1960).

\section{Disinfection of the Urethra}

This procedure was studied in gynaecological patients who were catheterized intermittently after operation. The patients were admitted, without selection, to two adjacent wards ( $D$ and $F$ ), which were administered as one by the same nursing staff. The number of catheterizations per patient. which varied from 5 to 59 (average 22), was almost identical in the two wards.

Two disinfectants were studied: (a) hydrargaphen (" penotrane ") urethral jelly, which contained phenylmercuric dinaphthylmethane disulphonate $(0.05 \%)$ and a local anaesthetic, and $(b)$ chlorhexidine digluconate (" hibitane ") $0.5 \%$ in a mixture of polyethylene glycols

*Working with a full-time grant from the Medical Research Council. 